\title{
Geschichtliche Lage und Ergebnisse der slowenischen Kunst
}

\author{
MiLČEK KOMELJ \\ (Ljubljana)
}

Der Schwerpunkt dieser Ausführungen ist als eine übersichtliche historische Darstellung der slowenischen Kunst und ihrer charakteristischen Erscheinungen gemeint. Dabei wird der Geschichte der Interpretationen, die diese Kunst mit dem spezifischen Begriff des Slowenentums zu verbinden versuchen, besondere Aufmerksamkeit gewidmet. Da diese Kunst durch Jahrhunderte in die westeuropäische Kultursphäre eingegliedert war und viele ihrer bedeutsamsten Repräsentanten nicht geborene Slowenen waren, dabei aber die bildende Kunst ,als solche“ nicht national bestimmt, sondern universal ist, so stellte sich nach der Erweckung der Nationalbewusstseins im 19. Jahrhundert und mit der Formierung der Kunstgeschichte auch die Frage, inwiefern in dieser Kunst auch die slowenischen Spezifika zu unterscheiden seien und welches die Beziehung zwischen der slowenischen Kunst und der Kunst auf dem slowenischen Territorium sei.

Das Bewusstsein einer slowenischen bildenden Kunst, die als Ausdruck der Existenz und Eigentümlichkeit der slowenischen Nationalität gesehen werden könnte, stammt aus der Zeit nach Ende des 19. Jahrhunderts und war mit der zunehmenden qualitativen Europäisierung der slowenischen Kultur im Zeichen der sogenannter Moderne verbunden. Diese Moderne gilt in der slowenischen Kultur als eine „Sternstunde“ auf allen Gebieten der künstlerischen Kreativität. Der erste slowenische Laienkünstlerverein (Slovensko umetniško društvo [Slowenischer Künstlerverein] genannt) versuchte damals, mit seiner Ausstellungstätigkeit auch auf die bildende Kunst als eine Legitimation der slowenischen nationalen Affirmation hinzuweisen. Diese Affirmation sah man früher vor allem in Verbindung mit der Literatur, basierend auf der slowenischen Sprache, durch welche wir uns als Slowenen bestimmt fühlten. In diesem $\mathrm{Zu}-$ sammenhang zeigte sich zum ersten Mal die künstlerische Qualität der sogenannten slowenischen Impressionisten, die aber in der Tat mehr postimpressionistisch und symbolistisch orientiert waren. Sie waren Autoren der ersten Gemälde, vor allem Landschaften, die im slowenischen Nationalbewusstsein mythische Attribute einnahmen: zum Beispiel Der Säer, ein Gemälde von Ivan Grohar, in dem eine symbolische Erscheinung der nationalen Hoffnung gesehen wurde. Diese Maler haben durch ihr Empfinden der zentralslowenischen 
subalpinen Natur die sogenannte "Stimmung“ in die Kunst hineingetragen. In dieser Stimmung könnte nach der Meinung einiger Kunsthistoriker (z. B. Nace Šumi) noch heute ein wissenschaftlich nur schwer zu bestimmender Nationalausdruck geahnt werden, der leichter zu fühlen als zu beschreiben wäre. Die Zeit um die Wende zum 20. Jahrhundert war auch eine Ära der ersten öffentlichen Standbilder, die einigen hochgeschätzten Persönlichkeiten der slowenischen Kultur aufgestellt wurden, z. B. den Dichtern Valentin Vodnik und France Prešeren sowie auch dem in deutscher Sprache schreibenden Polyhistor Valvasor. In derselben Zeit blühte in der Architektur der Sezessionstil, neben ihm aber auch der ausklingende Späthistorizismus. Die Wahl historischer Bauformen würde dabei, besonders wenn symbolisch repräsentative Gebäuden im Spiel waren, oft nach dem Kriterium des fiktiven „Nationalcharakters“ dieser Formen getroffen (so entstand das „Nationalhaus“, die heutige Nationalgalerie, in Ljubljana nach Mustern der Architektur aus Prag und die repräsentativen Gebäude in der slowenischen Steiermark nach deutschen Vorbildern). Die Altersgenossen der Impressionisten, die in Wien geschulten, mehr volkstümlichen Maler Vesnani (nach dem Künstlerverein Vesna genannt), die ihre Motivik aus dem folkloristischen Inventar schöpften, entschlossen sich aber aus Widerstand gegen die Germanisierung, ihren „Nationalausdruck“ bewusst zu suchen. Sie verbanden die Wiener Sezession mit der folkloristischen Volkstümlichkeit und popularisierten durch die Pflege der speziellen Gattungen des Kunstdesigns (Illustration, Postkarten) die Kunst auch unter den breiteren Schichten der Bevölkerung. Aber gerade die „impressionistische“ Kunst, die nicht durch solche volkstümlichen Begrenzungen blockiert war, sondern in ihrer Intensität als europäisch und im Kontext ihrer Zeit als modern angesehen werden kann, war jene, die im späteren slowenischen Kulturbewusstsein einen ganz auserlesenen nationalen Status erwarb. Mit ihr kam aber auch die Malerei als jener künstlerische Bereich zur Geltung, mit dem der Begriff unserer bildenden Kunst zuerst identifiziert wurde und der in unserem Bewusstsein der populärste ist. Da das slowenische Milieu sehr zurückgeblieben und nur für den narrativ-illustrativen Realismus empfänglich war, versuchten die impressionistischen Künstler, um auch von slowenischen Kritikern anerkannt zu werden und sich so in Slowenien überhaupt eine Existenz zu ermöglichen, zunächst Anerkennung im breiteren europäischem Kontext zu erreichen. (Nachdem sie in iher Heimat als „Fremde“ ausgestoßen worden waren, hatten sie als Klub Sava im Jahr 1904 eine Ausstellung bei Miethke in Wien.) So haben sie eine Kulturstrategie erarbeitet, die auch für spätere Generationen gültig blieb: auch unsere Besonderheiten sollte zuerst der Blick aus dem Ausland würdigen.

Unter solchen Umständen war Rihard Jakopičc, der organisatorische Protagonist der Impressionisten und Initiator der ersten slowenischen Kunstgalerie (in Ljubljana), sogar überzeugt, dass eine wahre slowenische bildende Kunst erst mit seiner Generation, d. h. mit den Impressionisten, begonnen habe. Um diese Überzeugung zu bestätigen, organisierte er im Jahr 1910 in Ljubljana die erste historische Retrospektive der slowenischen Malerei (mit dem Titel: 80 
Jahre der slowenischen Malerei). Dieser Ausstellung folgte im Jahr 1922 eine historische Retrospektive, die schon von Kunsthistorikern organisiert wurde. Ein Resultat dieser Ausstellung war auch, dass erstmals die Fragen nach dem Status der slowenischen Kunst gestellt wurden.

Infolge solcher Fragen und Diskussionen hat France Stelè, der vor dem Ersten Weltkrieg Konservator für das österreichische Land Krain (das heutige zentrale Slowenien) war, ein Buch mit dem Titel Abriss der Geschichte der Kunst bei den Slowenen geschrieben. Dieses Buch ist die erste vollständig konzipierte historische Darstellung der slowenischen bildenden Kunst auf einer wissenschaftlichen methodologischen Grundlage. Stelè hat angedeutet, dass auch die Kunst der älteren Zeiten als slowenisch zu charakterisieren sei. Infolge seines historisch bedingten ethnozentrischen Ausgangspunktes hat Stelè seine Darstellung gerade als einen Überblick der historischen Entstehung des slowenischen Kunstschaffens konzipiert. Er zeigte besonderes Interesse für das „Beheimaten“ der europäischen Impulse, die bis zum volkstümlich populären folkloristischen Niveau reichten. Die Produktion auf diesem Niveau stammte am evidentesten von den Einheimischen und so war sie am stärksten als mit der Bevölkerung „,verschmolzen“ erkennbar. Deshalb hat Stelè zum Beispiel das künstlerische Inventar der Burgen nicht in seine Darstellung aufgenommen; für ihn war nämlich diese Kunst als Produktion von Fremden ohne wahre Verbindungen mit der slowenischen Bevölkerung. Andererseits hat er aber einigen Erscheinungen, in denen die unmittelbare Verbindungen zwischen der Kunst und der slowenischen Bevölkerung bzw. ihrer Lebensrealität erkennbar waren, seine ganz besondere Aufmerksamkeit gewidmet (zum Beispiel dem qualitativ bescheidenen spätbarocken Maler Leopold Layer, der als Autor des bekanntesten slowenischen religiösen Gnadenbildes Mariahilf am Wallfahrtsort Brezje noch heute populär ist). Obwohl Stelè die slowenische Kunst als einen vorwiegend qualitativ bescheideneren Ausdruck der Aneignung der Stile auch in der Produktion der Vergangenheit erkannt hatte, bemühte jedoch auch er den Begriff des in der bildenden Kunst zu erkennenden "Slowenentums“ ausdrücklich erst mit der Erweckung des Nationalbewusstseins im 19. Jahrhundert und vor allem mit der Erscheinung des sogenannten Impressionismus. Infolge dieser ethnozentrischen Gesinnung eröffneten sich mit dem Studium unserer Kunst von Anfang an als etwas Wesentliches auch Fragen nach dem Verhältnis zwischen der Kunst auf dem slowenischen Territorium und der slowenischen Kunst, da ein Großteil vor allem der qualitativ hervorragenden Produktion selbstverständlich mit Auftraggebern und Künstlern, die aus dem Ausland stammten bzw. nicht als Slowenen geboren wurden, verbunden war. Seitdem versuchten die Autoren, die über die Kunst schrieben, auch deren spezifischen Eigenschaften zu finden. Sie haben diese Eigenschaften sowohl in den Charakterbestimmungen (vor allem im sogenannten Lyrismus) als auch in einigen Prinzipien der Stiladaptierung gesucht. Eine solche Adaptierung war besonders charakteristisch gerade für die „Impressionisten“, die die historisch "retardierte» impressionistische Form dem Ausdruck einer ganz modernen Stimmung an- 
passten. Das Erkennen solcher Anpassungen alter Mittel an einen aktuellen Inhalt schuf mit der Zeit einen hartnäckigen Komplex wegen des im Wesentlichen zurückgebliebenen Charakters der slowenischen Kunst (weil das slowenische Territorium die europäischen Kunstrichtungen fast immer mit einer Retardation aufnahm). In diesem Kontext hat besonders Nace Šumi eine These von der Anpassung der traditionellen Stilbegriffe an den jeweils aktuellen Inhalt entwickelt.

Die Bezeichnung „Kunst auf dem slowenischen Territorium“ hat für die älteren Kunstperioden in der Kunstgeschichte ihre Geltung behalten, aber mit der Zeit ist auch die Erkenntnis der Notwendigkeit ins Bewusstsein gedrungen, alle Kunst auf diesem Territorium ohne Rücksicht auf die Nationalität und die soziale Klassenzugehörigkeit gleichwertig zu behandeln. Dieselbe historische Logik hat auch die Tendenz diktiert, dass einerseits die Kunst in diesem Kontext von slowenischen Kunsthistorikern bei der Erforschung verschiedener Kunstperioden dem jeweiligen historischen, mit Slowenen besiedelten Territorium gemäß zu behandeln sei: dieses Territorium wurde nämlich durch die Jahrhunderte hindurch allmählich drastisch verkleinert. Andererseits sind aber mit dem Begriff ,,slowenische Kunst“, wenn es um eine Darstellung der künstlerischen Kreativität der Slowenen geht, auch die Werke der im Ausland tätigen Künstler slowenischer Herkunft gemeint. Diesen Künstlern wurde lange keine besondere Aufmerksamkeit gewidmet, da sie keinen bemerkbaren Einfluss auf die Tätigkeit auf dem slowenischen Territorium ausgeübt hatten (so zum Beispiel der neoklassizistische Maler Franc Kavčič (Franz Caucig), der Rektor der Wiener Akademie war, der Münchner Hofmedailleur Šega, der Architekt von Minneapolis Ivan Jager, vor dem Ersten Weltkrieg in Amerika der Maler Gregor Perušek bzw. Harvey Gregory Pruscheck); in bezug auf die neuere Kunst hat dabei aber auch das negative Verhältnis gegenüber der politischen Emigration das Seinige beigetragen (unter den schon vor dem Krieg ausgewanderten Slowenen zeichnete sich der in Australien tätige Stanislav Rapotec aus und unter den Emigranten nach dem Zweiten Weltkrieg der weltberühmte, in Paris tätige Zoran Mušič; der symbolisch ansehnlichste öffentliche Auftrag aber wurde dem Jesuiten, Theologen und Maler Marko Ivan Rupnik, dem Autor von Mosaiken in der Privatkapelle des Papstes Johannes Paul II. im Vatikan, zuteil).

Die Kunst der im Ausland geborenen Künstler und die mit fremden Auftraggebern verbundene Kunst, die vormals als „fremd“ bezeichnet wurde (sowie auch die importierten Kunstdenkmäler) wird heute von den Forschern zumeist als „europäisch“ bezeichnet bzw. ihre „Fremdartigkeit“ in Anführungszeichen gesetzt. Eine ganz sachliche, wissenschaftlich unideologische, oder besser gesagt: unengagierte Einsicht in die künstlerische Produktion, wie sie in der Kunstgeschichte nach dem Zweiten Weltkrieg formiert wurde, wird nämlich mehr den künstlerischen Verbindungen im europäischen bzw. regionalen Kontext als der nur schwer bestimmbaren, die nationale Eigentümlichkeit bestätigenden slowenischen Besonderheiten zugeschrieben. So sind einige, 
z. B. das Akademiemitglied Emilijan Cevc, der in seiner Jugend mit intuitiver Sensibilität und dichterischem Sprachausdruck gerade für solche nationale Dimensionen empfänglich war, heute, nach langjährigen Forschungen, immer mehr der Ansicht, es gäbe gar keine spezifische slowenische Kunst, da die slowenischen Spezifika nicht wissenschaftlich zu bestimmen seien; natürlich könnten wir in diesem Sinn auch nicht von einer deutschen oder französischen Kunst sprechen. Auch in der Frage der slawischen (bzw. der sogenannten „slawistischen“) Kunstgeschichte, wie sie von dem in Polen tätigen slowenischen Kunsthistoriker Vojeslav Molč konzipiert wurde, zeigt sich, dass eine solche Konzeption nicht etwa in slawischen Charakteristiken bzw. Besonderheiten der Kunst begründet liegt, sondern sie setzt eine historisch bedingte Verschiedenheit bei verschiedenen slawischen Nationen voraus. Eine solche sachliche, historisch begründete Ansicht folgt natürlich viel leichter den regional spezifischen als den vermuteten nationalen Besonderheiten, also den Charakteristiken der Kulturkreise, die auf einem gemeinsamen zivilisatorischkulturellen Territorium auch verschiedene Nationen verbinden können. Eine solche Ansicht hat sich dank Stelè und seiner Nachfolger in Slowenien als die grundlegende kunsthistorische Methodologie unter dem Einfluss der deutschen sogenannten Kunstgeographie durchgesetzt, auch als Folge der sehr spezifischen geographischen Lage des heutigen Slowenien im Knotenpunkt verschiedener Einflüsse, Anknüpfungen und Durchdringungen des nördlichen und des mediterranen Kulturraums, aber auch der germanischen, romanischen, slawischen und ungarischen Nationalitäten. (Ein Musterbeispiel dieser Gesinnung ist das Werk Die Kunst im Küstenland von Stelè, in dem die Interaktion der sogenannten nördlichen und mediterranen kulturgeographischen Zonen lehrreich dargestellt wird.) Und diese geographische Gegliedertheit gilt vorwiegend sogar als die Hauptcharakteristik und Hauptdeterminante unserer gesamten Produktion in der bildenden Kunst. Ihre Phänomene und Relationen waren auch mit der spezifischen historischen Lage eng verbunden, die nach der Christianisierung mit der für kulturelle Einwirkungen sehr bedeutenden Beteiligung der kirchlichen Herrschaft auf dem heutigen slowenischen Territorium zwischen Aquilea und Salzburg im Jahr 811 determiniert war, sowie auch mit der jahrhundertelangen Unterwerfung verschiedener nichtslowenischer Gewalten nach dem Untergang des ersten slowenischen Staates Karantanien am Anfang des 11. Jahrhunderts, dem erst im vorigen Jahrhundert zunächst eine Republik innerhalb der jugoslawischen Föderation und dann im Jahr 1991 der selbstständige Staat Slowenien folgten. Zur Profilierung und qualitativen Verschiedenartigkeit der Kunstdenkmäler haben - oft entscheidend - auch die Macht und die kulturelle Orientierung der Auftraggeber beigetragen; diese haben die Kunst und vor allem die Architektur mit ihrer Beschlagenheit und ihren Kulturverbindungen stark geprägt, so wie es zum Beispiel in der Zeit der Gotik die Grafen von Cilli taten.

Die ersten Erkenntnisse über die Kunst auf dem slowenischen Territorium wurden schon früher im Rahmen der im 17. Jahrhundert entwickelten Hei- 
matkunde und vor allem in der Zeit des Historizismus im 19. Jahrhundert gewonnen. Aber erst als Resultat der Kunstgeschichte des 20. Jahrhunderts hat sich, ausgehend von der letzten Jahrhundertwende, eine Sicht auf die historische Lage und die Ergebnisse dieser Kunst weit in die Vergangenheit eröffnet; diese Sicht deutet mit noch ungewissen, tastenden Vermutungen auch in die Zukunft.

Wenn wir nur die Geschichte der bildenden Kunst auf dem slowenischen Territorium erkennen wollten, dann müssten wir eine besondere Bedeutung dieser Kunst bereits in der Vorgeschichte und der Antike betonen: oft kann eine Kontinuität der Besiedlung von der Antike oder sogar der Vorgeschichte bis zur heutigen Zeit erwiesen werden. Auch das mittelalterliche Ljubljana würde in der Nähe der antiken Stadt Emona, die im heutigen Stadtzentrum gelegen war, gegründet. In der vorhistorischen Kunst zeichnet sich vor allem die sogenannte Kunst der Situlen aus und von den römischen antiken Kunstdenkmälern die monumentale Nekropole in Šempeter. In Verbindung mit Slowenen als Kulturträgern zeichneten sich aber zuerst vor allem die Denkmäler aus, die in der antiken Tradition den Wert eines kulturellen Statussymbols hatten, so z. B. der „Fürstenstein“ und „Herzogsthron“ im heutigen österreichischen Kärnten (Kärnten war im frühen Mittelalter das politische Zentrum des slowenischen Territoriums) oder einzelne vorromanische Reliefe, die besondere Rätsel für die Interpretation darstellen, so z. B. ein Relief in Hodiše (Kärnten), das in der Tradition der altertümlichen Verhältnisse zu Sternbildern gedeutet wird. Mit der Bildung der späteren sogenannten historischen Stile wurden slowenische Länder selbstverständlich der europäischen bzw. - mit Ausnahme des Küstenlandes, das ausgesprochen italienisch, venezianisch geprägt war - vor allem „mitteleuropäischen“ geschichtlichen Strömung (und deshalb hat sie Jakopič als deutsch beeinflusst angesehen) einverleibt und waren auch durch die künstlerischen Einflüsse am meisten mit den naheliegenden kirchlichen bzw. politischen Zentren verbunden.

Als nach außen hin augenfälligste und allgemeinste slowenische Charakteristik können wir eine große Anzahl von den auf den slowenischen Hügeln zerstreuten Kirchen bemerken, die als ein besonderes, christlich geprägtes Merkmal in der Landschaft stehen. Die Lokationen dieser Kirchen sind oft durch die Kontinuität der älteren heidnischen Heiligenstätten bedingt. In der späteren kreativen Kontinuität sind in den slowenischen Ländern neben vielen typischen, aber vorwiegend nur mittelmäßigen Denkmälern auch einzelne Denkmäler entstanden, die Zeugnis von der einstigen mittelalterlichen, in einzelnem Fällen auch ziemlich großzügigen Eingliederung der Kunst auf dem slowenischen Territorium in breitere kulturelle Verhältnisse und Verbindungen ablegen; sie sind nur im Kontext eines kulturellen und als geistiges Ganzes zu begreifenden Europa und als Ausdruck weit ausgebreiteter Beziehungen erklärbar. Dieses gilt für die ersten monastischen monumentalen Gebäude in der Zeit der Romanik, als eine Europäisierung zum ersten Mal auch auf dem Gebiet der Kunst, vor allem der kirchlichen Architektur genau rekonstruiert 
werden konnte. Diese ist in den meisten Fällen unter den späteren Umbauten versteckt geblieben, oft ist sie jedoch wenigstens in den Grundrissen dokumentiert. Deswegen hat sich Akademiemitglied Marijan Zadnikar, der für uns die Zeit der Romanik entdeckte, vor allem der Methodologie der typologischen Darstellung gewidmet, die mit ihrer geographisch gegliederten Buntheit Slowenien deutlich als ein Land der Übergänge aufzeigt, da wir auf diesem Territorium verschiedene geographisch bedingte Typen der Architektur antreffen, die am klarsten in den Standardformen der mediterranen Apsis und im Typus des nördlichen Presbyteriums mit dem anknüpfenden sogenannten Ostturm zu erkennen sind. Ebenso ist, wahrscheinlich wegen der Entfernung von den Kulturzentren, das zentrale romanische Denkmal, die Kirche des Zisterzienserklosters in Stična (Sittich) typologisch von europäischer Bedeutung, da sie als ein Beispiel des alten vorbernardinischen, noch cluniazensichen Bautypus eine Ausnahme in der Zisterzienserarchitektur bedeutet. Von internationaler Bedeutung sind auch die dort entstandenen illuminierten Kodices, die als Ausdruck der Fluktuation bzw. verschiedener Verbindungen der Illuminatoren und Schreiber interpretiert wurden. Die Kartause Žiče (Seiz) zeigt mit ihrem Aussehen sogar einen direkten Impuls seitens der französischen Architektur, der aus Frankreich ohne deutsche Vermittlung kam; so gilt das Hauptportal der Kirche im näherliegenden Špitalič (der vormaligen unteren Kirche der Kartause) sogar als das älteste Beispiel der Knospenkapitelle in einstigen deutschen Ländern. Noch ausdrücklicher zeigt sich die Lage der slowenischen Länder in den Jahrhunderten der Gotik, die das slowenische Territorium ganz „eroberte", die Städte urbanistisch bestimmte und dieses Territorium bis zum heutigen Tag augenfällig prägte. Auch die Meisterwerke dieser Kunst stehen im Kontext der Verbindungen, die von den monastischen oder aristokratischen Auftraggebern abhingen, z. B. die Zisterzienserkirche in Kostanjevica an der Krka (daneben können wir heute einen später erweiterten monumentalen Arkadenhof des Klosters bewundern) oder die Kirchen in Ptujska gora und Hajdina, wo die aristokratischen Kulturverbindungen eine vornehme bildhauerische Kunst der Parler zu uns gebracht haben.

Sonst sind bei uns vornehmlich die Prozesse des „Beheimatens“ der europäischen Konzeptionen mit stark regionalen Akzenten charakteristisch. Stelè erklärte z. B. den Typus des kirchlichen Saalraums im 15. Jahrhundert als einen regionalen Ausdruck der "Sondergotik“ und sah besonders die kirchliche Wandmalerei in ihrer Eingliederung in den Kulturraum der sogenannten subalpinen Zone zwischen dem Golf von Quarnero und den Alpen als eine Sondererscheinung an. Wegen der für Krain charakteristischen ikonographischen Konsequenz hat er sogar den spezifischen Terminus „Krainer Presbyterium“ eingeführt. Als eine bedeutende Erscheinung ist auch die Malerei des Malers Janez (Johannes) Aquila (und seiner Mitarbeiter) im Grenzgebiet Sloweniens, Österreichs und Ungarns zu erwähnen (mit den Selbstporträts des Malers, die manchmal als die ersten Selbstporträts der europäischen Wandmalerei bezeichnet werden), die das kulturelle Patrimonium dieser drei Staten verbindet. 
Sonst reicht die stilistische Spannweite der gotischen Malerei in Slowenien von italienischen Impulsen mit der spezifischen Rolle der sogenannten reisenden „friaulischen Maler“ (die in Görz residierten) bis hin zu deutschen Einflüssen, besonders durch die damals sehr verbreiteten graphischen Vorbilder, da vor allem durch die Werke des Meisters E. S.; einzelne Werke wurden auch von reisenden deutschen Malern angefertigt. Wegen seiner ein wenig naiven Expression ist die Wandmalerei von Andreas aus Otting im Kirchlein des Hl. Geistes in Slovenj Gradec im Slowenischen Kärnten besonders erwähnenswert. Die lyrischen Komponenten der aus Kärnten stammenden und im Sinn des retardierten „weichen Stils“ in Krain heimisch gewordenen Malerei des Janez Ljubljanski wurden aber später, im 20. Jahrhundert, etwas mystifizierend gar als ein Beispiel des slowenischen lyrischen National- bzw. Regionalcharakters interpretiert. Slowenischer Nationalität war der bedeutendste Maler im (heute österreichischen) Kärnten Tomaž Beljaški (Thomas von Villach bzw. Tomaž Artula). In diese spätmittelalterliche regionale Kultur interpolierten sich nach der Wende zum 16. Jahrhundert auch die Komponenten der Renaissance; in diesem Kontext sind die auch ikonographisch sehr reichen Wandmalereien in der Kirche der Heiligen Primus und Felizian oberhalb Kamnik von besonderer Qualität.

Damit stellt sich als eine der Grundfragen unserer Kunstgeschichte die Frage nach der Existenz und Rolle der Renaissance in Slowenien, eine Frage, die noch heute sehr verschieden beantwortet wird. In bezug auf die bildende Kunst ist (ungeachtet der kulturellen Bedeutung der slowenischen Reformation mit dem großen Reformator Primož Trubar, der auch den Begriff ,die Slowenen" in seiner kulturell verbindlichen Rolle in seinen Büchern in Umlauf brachte) das Verhältnis zur Renaissance beinahe als einer der Komplexe der slowenischen Kultur zu begreifen (ähnlich haben Slowenen den anderen südslawischen Nationen aus Ex-Jugoslawien manchmal eine zu schwache Erfahrung der Aufklärung als Mangel vorgeworfen). Im 16. Jahrhundert ist einerseits eine Anpassung der alten kirchlichen gotischen Bauformen an eine neue, mehr profane Stimmung bemerkbar; so bestand in der Kunstgeschichte ein Dilemma über das Verhältnis der ausklingenden Gotik zu der sich gerade anbahnenden Renaissance. Die ausdrücklichsten italienischen Renaissanceformen mit der Burgarchitektur im gesamten slowenischen Territorium (die auf etwas niedrigerem Niveau auch in der Architektur in den Städten sowie in der Architektur der Festungen, die von der Bevölkerung als Abwehr drohender türkischer Angriffe gebaut wurden) stammten von italienischen Architekten, Baumeistern und Steinmetzen als eine Folge der Fortifikationsplanung der slowenischen Länder wegen der Türkengefahr; so ist ihre Entstehung nicht etwa vor allem mit einer kulturellen Umorientierung oder dem Humanismus in Verbindung zu bringen. Der einzige „Renaissancehof“ in Slowenien war mit den kulturellen Anstrengungen des Bischofs von Ljubljana und Diplomaten Krištof Ravbar (Rauber) verbunden. Ravbar hat den Bildhauer Osbolt Kittel aus Deutschland (Augsburg) zu sich eingeladen. Kittel war Autor von 
sehr bedeutenden Epitaphen der Bischöfe mit ihren ganzfigürlichen Porträts in der bischöflichen Residenz Gornji Grad. Sonst kam die Renaissance in Slowenien eher in formalen Standardcharakteristiken (vor allem in den meistens reliefartigen Epitaphen) als etwa in der geistigen Gesinnung einer Wiedergeburt der Kunst zum Ausdruck. Der geistig außerordentlich rege und revolutionäre slowenische Protestantismus bediente sich für die slowenischen Bücher (vor allem für die Übersetzung der Bibel durch Jurij Dalmatin, 1584) der graphischen Illustrationen aus dem Repertoire der deutschen Holzschnitzer und ist sonst in der slowenischen Kunst meistens im Widerhall der spezifischen Ikonographie erkennbar. (Das bedeutendste Beispiel dafür sind die Wandmalereien im Stil der italienischen Spätrenaissance im sogenannten „Lutheranischen Keller" in Sevnica.)

Die Rolle der Renaissance im Sinne einer kulturellen Wiedergeburt übernahm im Bereich der bildenden Kunst nach der Wende zum 18. Jahrhundert erst das Barock. Die Kunst der Periode zwischen Gotik und Barock, als im Zeichen der katholischen Restauration einige auch später aktuelle kirchliche Bautypen, der Typus eines länglichen Schiffs mit den Kapellen (die Jesuitenkirche in Ljubljana), der zentrale (Nova Štifta) und auch der zentralisierte Typus, eingeführt wurden, während in der Bildhauerei als zentrale slowenische Spezifik die sogenannten ,goldenen Altäre“ vorherrschten, hat einen Übergangscharakter behalten. Infolgedessen wird in einer Kunstgeschichte die Kunst dieser Periode lieber als mit der Bezeichnung einer nichtausgeprägten Mischung der ausklingenden Gotik, der Renaissance, des Manierismus und des angehenden Barock nur mit der Zeit ihres Entstehens als die Kunst des 16. und des 17. Jahrhunderts bezeichnet. Wegen der vorherrschenden qualitativen Bescheidenheit dieser Produktion wurde die Suche nach einer Stilbestimmungen, mit der unsere Kunstgeschichte heute noch beschäftigt ist, mehr auf die Identifizierung der Elemente des Schmuckrepertoirs (vor allem der für die Zeit charakteristischen Ornamente) als auf die Kriterien des Aufspürens von Konzepten und geistigen Inhalten gegründet. Als Initiator der kulturhistorischen Unternehmen, darunter auch der ersten künstlerischen Publikationen (z. B. eines Buches der graphischen Illustrationen nach den Ovidischen Metamorphosen), hat sich mit seiner graphischen Werkstatt in jener Zeit der Polyhistor Johann Weichard Valvasor ausgezeichnet, neben ihm jedoch auch einige adlige Auftraggeber, welche die sogenannten ,fremden Meister“, unter ihnen reisende Deutsche und Holländer, bezahlten. Der bedeutendste auch in Slowenien tätige Maler dieser Periode war der belgische, in Italien ausgebildete Meister Almanach. Diese und auch andere, nur importierte Werke der europäischen Meister wurden erst unlängst in Zusammenarbeit der Nationalgalerie Ljubljana mit dem italienischen Experten Federico Zeri gründlicher oder aufs neue historisch bewertet.

Das Barock hat sich nach den verdienstvollen Anfängen der Academia operosorum in Ljubljana, Vorfahre der heutigen Slowenischen Akademie der Wissenschaft und Kunst (Vorfahre der ersten Academia war freilich die aristokratische religiöse Bruderschaft des Hl. Dismas), in Bewunderung der 
se Bruderschaft des Hl. Dismas), in Bewunderung der altrömischen und der zeitgenössischen italienischen Kultur intensiv an italienischen Vorbildern orientiert, vor allem an der venezianischen Kultur, aber auch direkt an Rom (der Architekt der barocken Domkirche in Ljubljana war Andrea Pozzo); die schwungvolle patriotische Rhetorik operierte damals sogar mit der Metapher „Ljubljana als eines neuen Rom“. Erst nach der ersten Hälfte des 18. Jahrhunderts wandte sich die Kunstorientierung, auch infolge des kaiserlichen Dekrets über eine neue Regelung von Gesellen-Wanderungen der angehenden Künstler, den deutschsprachigen Ländern zu. Ljubljana ist erst in dieser Zeit zu einem bedeutenden Zentrum der bildenden Kunst geworden, das aus Italien schöpfte, ausländische Künstler akzeptierte und seine Einflüsse auch über die Grenzen des Herzogtums Krain und des slowenischen Territoriums hinaussandte, besonders nach Kroatien. Die Kunst der Italiener, die die Architektur von Ljubljana prägten, wurde auch in der Provinz heimisch. Der Barockstil hat bis zur heutigen Zeit die slowenische, mit Kirchen und Kapellen besäte Landschaft am stärksten geprägt und ist in die künstlerische Tätigkeit aller sozialen Schichten gedrungen. Der Lyriker Tomaž Šalamun hat dazu sogar den Vers „Barock, die Nahrung der Nation" gedichtet.

Unter den während der Blüte des Barock entstandenen Architekturdenkmälern zeichnet sich die Ursulinenkirche in Ljubljana mit ihren rein architektonischen Qualitäten in der Tradition des norditalienischen palladianischen Klassizismus aus. Die illusionistischen Malereien auf den Gewölben der Domkirche von Ljubljana sind das bedeutendste künstlerische Ergebnis des friulanischen Malers Giuglio Quaglio (der auch der Autor der etwas späteren Malerei auf dem Gewölbe des Saals der ersten slowenischen öffentlichen Bibliothek im Seminarpalast von Ljubljana - war) und haben eine reiche und auch in der Provinz verbreitete Tradition der slowenischen illusionistischen Malerei initiiert. Die Nachfrage nach kirchlichen Bildern hat den lothringischen Maler Valentin Metzinger angezogen; später, im ausklingenden Barock, wirkte für die wichtigsten bischöflichen und monastischen Auftraggeber auch Johann Martin Schmidt, Kremser-Schmidt genannt, welchen wahrscheinlich der Jesuit Gabriel Gruber in Slowenien eingeführt hatte. Kremser-Schmidt hat im Gruber-Palast in Ljubljana (in dem sich auch ein spätbarockes Treppenhaus von außenordentlichem Raffinement befindet) die Kapelle bemalt, die für eines seiner Hauptwerke gehalten wird. Die zentrale künstlerische Persönlichkeit der Barockzeit, der Bildhauer Francesco Robba, kann als für der bedeutendste Bildhauer seiner Zeit zwischen Wien und Venedig angesehen werden. Sein künstlerisches Profil und der Schwung seines individuell geprägten Schaffens sowie auch das Schaffen anderer einheimischen Künstler war teilweise an die Kulturerwartungen des Millieus gebunden; so wurden diese Künstler wegen ihrer Einbindung in den Kontext unseres Kulturbewusstseins selbstverständlich zu den slowenischen bzw. krainischen, d.h. „heimischen“ Künstlern gezählt. Unter den slowenischen Protagonisten war aber der in Rom geschulte änigmatische Maler Fortunat Bergant der bei weitem bedeutendste. Bergant war 
ein Porträtist höchsten Ranges und auch Autor von genrehaft geprägten expressiven religiösen Bildnissen mit betontem menschlichem Leidensausdruck. Deswegen wurde er für den ,am meisten slowenischen“ unter den Barockmalern gehalten; wegen seiner Ausdruckskraft wurde er aber als der einzige Protagonist des Barock auch von den Malern des 20. Jahrhunderts als aktuell empfunden, vor allem von Rihard Jakopič und Gabrijel Stupica, der ihn einmal sogar ,unseren Goya“ genannt hat). Das erste in Europa allgemein bekannte Gemälde aus Slowenien, Der Tod des Hl. Franziskus Xaver (ein Werk von Franz Michael Reinwaldt) in der Wallfahrtskirche Straže bei Gornji Grad, das z. B. auch für Prag und Dresden kopiert wurde, erreichte seinen internationalen Ruhm infolge seiner angeblichen Wunder vollbringenden Eigenschaften und nicht seiner künstlerischen Bedeutung wegen.

Ein anderer Impuls des italienischen Barock erreichte Slowenien durch die Vermittlung Österreichs, vor allem in der slowenischen Unter-Steiermark, die sich an ihrem Landeszentrum Graz orientierte. Für die Kunst, besonders für die Malerei, waren die aristokratischen Auftraggeber von allergrößter Bedeutung. Ein besonders wichtiger Aufraggeber war der Besitzer der vornehmsten Burgen dieser Gegend, Graf Ignaz Maria Attems; für ihn wirkte der österreichische Maler Ignaz Maria Flurer, unter den slowenischen Künstlern hat sich in Österreich sein „Hofmaler“ Frančišek Mihael Remb (in der österreichischen kunsthistorischen Literatur Remp) durchgesetzt. Nach Mitte des 18. Jahrhunderts wurde mit dem monumentalen Bildhauer Jožef (Josef) Straub auch Maribor (damals Marburg) zu einem Kunstzentrum.

Für das Küstenland war (mit Ausnahme von Koper, Isola und Piran, die damals Bestandteil der venezianischen Republik waren), ungeachtet der Nähe zu Italien, auch in der Barockzeit die tektonische Ruhe charakteristisch, in der ein Widerhall der Antike lebendig blieb, die auch in der Urbanität des Karstes zu erkennen ist.

Über solche allgemeinen Regionalcharakteristika nachdenkend, hat Šumi als das Zentrum bzw. den Koordinierungspunkt von verschiedenen Impulsen gerade Ljubljana erkannt, wo er sogar eine ,slowenische Synthese“ sah, die alle Extreme verband und transformierte, vor allem die mediterrane Tektonik und die nördliche Pittoreskheit. Auch deswegen war aus der slowenischen Perspektive nach der Germanisierung Kärntens, das nach dem Ersten Weltkrieg infolge des Plebiszitsresultates an Österreich fiel, mit den Anfängen der slowenischen Kunstgeschichte die größte Aufmerksamkeit der Forscher eben auf das ehemalige Herzogtum Krain und vor allem auf Ljubljana als das sich allmählich entwickelnde Nationalzentrum gerichtet.

Das Barock klang mit seiner Werkstättentraditon noch weit ins 19. Jahrhundert hinein. In sein Erbe mischten sich allmählich auch die Formen des Biedermeiers bzw. der Nazarenerkunst. In der ersten Hälfte des 19. Jahrhunderts wurden die ersten akademisch geschulten profanen biedermeirlichen Maler tätig. In der Malerei des seinerzeit sehr populären Matevž Langus, der im kollektiven Bewusstsein der Slowenen eine betont nationale Bedeutung 
wegen seiner Freundschaft mit dem von ihm zwar nicht porträtierten Dichter France Prešeren einnimmt, können wir ein Beispiel des für das damalige Krain typischen bescheidenen künstlerischen Niveaus erkennen. Mit Prešeren ist im kollektiven Bewusstsein seiner Landsleute auch der Maler Franz Kurz zum Thurn und Goldenstein verankert, Autor des postumen Porträts von diesem in der slowenischen nationalen Mythologie so bedeutenden Dichter. Als Künstler zeichnete sich neben dem manchmal eher romantisch orientierten Mihael Stroj (Stroy) vor allem Jožef Tominc (Tominz) aus. Tominc war der wichtigste Porträtist im damals blühenden Triest. Das multinationale Triest war im 19. Jahrhundert ein großes Zentrum der Slowenen, die mit anderen slawischen Nationen um die Jahrhundertmitte auch an der politischen Macht teilzuhaben begannen; an der Wende zum 20. Jahrhundert zählte es sogar mehr slowenische Einwohner als Ljubljana. Triest war auch ein bedeutendes Zentrum der neoklassizistischen Architektur. (Sonst ist in Slowenien unter den seltenen Beispielen der neoklassizistischen Architektur die kosmopolitische Konzeption des Kurorts Rogaška Slatina zu erwähnen.) Mit seinem Sinn für individuelle Charakterisierung wurde Tominc zum Chronisten des auf Profit gerichteten geistigen Profils der Triester Bourgeoisie. Mit seinem individuellen Charakter eröffnete er in der Kunstgeschichte die Frage nach dem „Mitteleuropäertum“, da seine Malerei mit ihrer Ausgelassenheit und ihrem plastischen Realismus weder in die österreichische biedermeierliche noch in die italienische Kunst restlos eingereiht werden kann. In der biedermeierlichen Manier mit beigemengter Romantik ist auch die slowenische Landschaft zum ersten Mal ins Bewusstsein bildender Künstler deutlicher eingetreten, da vor allem - wie in der romantischen Dichtung Krst pri Savici (Die Taufe bei Savica) von Prešeren die alpine Landschaft Oberkrains mit seinen Seen und dem höchsten slowenischen Berg, dem Triglav; diese Landschaft malten die Maler sowohl slowenischer (Marko Pernhart) als auch deutscher Nation (Anton Karinger, der in Ljubljana wirkte).

Die Wiedergeburt der kirchlichen Malerei setzte im Zeichen der romantischen Anlehnung an die Nazarener und an die Ideale der italienischen Renaissance ein mit dem Maler Janez Wolf, einem Freund und Bewunderer von Anselm Feuerbach und Lehrer der Realisten Janez und Jurij Šubic sowie Anton Ažbe. Diese realistischen Maler haben in den letzten Jahrzehnten des 19. Jahrhunderts die slowenische Malerei wieder auf das europäische Niveau gehoben, waren aber gerade deswegen gezwungen, sich in das Kulturleben auBerhalb Sloweniens einzugliedern, wo sie bleiben mussten, um ihre nackte Existenz überhaupt retten zu können. Janez Šubic hat sich in Kaiserslautern niedergelassen, Jurij Šubic in Paris, die Malerin Ivana Kobilca für eine Zeitlang in Berlin und Anton Ažbe in München. Gerade diese zu früh verstorbenen Künstler waren in der Zeit der Konsolidierung des slowenischen Nationalbewusstseins sehr stark von einer als Gesamtheit zu begreifenden slowenischen Kultur überzeugt. Das kam auch in ihren Verbindungen mit Literaten zum Ausdruck, mit denen auch die Anfänge der slowenischen künstlerischen Bü- 
cherillustration zusammenhingen, vor allem in der von Josip Stritar redigierten, in Wien gedruckten Literaturzeitschrift Zvon (Die Glocke). Diese geistige Wechselwirkung wurde nach der letzten Jahrhundertwende in der Sensibilität der Dichter der sogenannten Moderne (Ivan Cankar, Oton Župančič) noch gesteigert. Cankar und Župančič haben nach der oben bereits erwähnten Ausstellung der Impressionisten in Wien im Jahr 1904 als erste ihre Malerei interpretiert und vertraten die Meinung, dass ihre spezifische slowenische Stimmung im (lyrisch melancholischen) Ausdruck und nicht etwa in der Evidenz oder gar im Folklorismus der Motive verwurzelt sei.

Unter den Realisten hat sich mit seiner weitreichenden pädagogischen Bedeutung Anton Ažbe ausgezeichnet. In seiner berühmten Schule in München lehrte er eine Reihe von späteren bedeutenden europäischen Malern, darunter Kandinsky, aber auch die Bahnbrecher der modernistischen Orientierung der slawischen nationalen Künste, so z. B. die sogenannten slowenischen Impressionisten. Wegen seiner internationalen Bedeutung hat er sich als eine einflussreiche kulturelle Persönlichkeit, wenn auch nicht wegen seiner eigenen Malerei, ins europäische und vor allem ins deutsche Kulturbewusstsein von allen slowenischen Künstlern am stärksten eingeprägt. Spätere slowenische Maler, teilweise schon die Expressionisten, vor allem aber jene die Angst- und Entfremdungsgefühle betonenden Maler nach dem Zweitem Weltkrieg, die manchmal auch als „Maler der Existenz“ bezeichnet wurden, haben aber unter den Realisten den Maler Jožef Petkovšek wegen seiner Vereinzelung, die mit seiner Geisteskrankheit verbunden war, als den einzigen aktuell gebliebenen Künstler (neben Bergant) gepriesen. Nachdem Petkovšek von Jakopič postum entdeckt worden war (durch dessen Aufnahme in die historische Ausstellung der slowenischen Malerei), hat er allmählich sogar die metaphorische Funktion eines ,slowenischen van Gogh“" gewonnen, die mit den stilistischen Charakteristika der Malerei von Petkovšek eigentlich nichts zu tun hat. Sein autobiographisches Bildnis Daheim, das zum ersten Mal von Ivan Cankar, der auch ein Landsmann von Petkovšek aus Vrhnika war, in literarischer Form interpretiert wurde, ist zum malerischen Symbol geworden, in welches die nachfolgenden Generationen ihr Empfinden des „Slowenentum-Traumas“ hineinprojizierten, während die Kunsthistoriker versuchten, an ihm ihre Forschungs- und Interpretationsverfahren zu erproben, um damit durch die formelle Oberfläche auch dem sensiblen geistigen Kern des leidenden Menschentums nahezukommen. Petkovšek war eine einsame, in die kunstgeschichtlichen Strömungen schwer einzureihende Erscheinung, da er sich vor allem ums formale akademische Können krampfhaft bemühte. Er hat den schmerzlichen Ausdruck seiner Malerei gerade mit der Unvollkommenheit seines realistisch orientierten künstlerischen Wollens erreicht, ohne dabei dilettantisch oder naiv zu wirken.

Sonst war die Malerei, die nicht mehr dem religiösen Universalismus, sondern subjektivem Empfinden und persönlichen Dilemmata zugewendet war, dazu gehörte um die Jahrhundertwende auch die Problematik des Nationalen, in der slowenischen Kunst von den „Impressionisten“ initiiert. Unter ihnen 
haben in ihren Werken die geistigen Dimensionen am persönlichsten der poetische Ivan Grohar und der ekstatisch dynamische Rihard Jakopič ausgedrückt. Dieser hat die modernen Richtungen - er hat sich in einzelnen Werken und Studien sogar der Abstraktion genähert - mit der malerischen Tradition des Barock verbunden. So ist der Anfang des 20. Jahrhunderts, für die slowenischen Kunsthistoriker der Anfang der als national bezeichneten slowenischen Kunst, auch ein Anfang des slowenischen Modernismus als eines modernen, subjektiv betonten und formal inventiven Kunstschaffens.

In diesem geistigen Kontext können wir auch die Architektur des Altergenossen der Impressionisten Jože Plečnik interpretieren. Plečnik hat nach der Meinung der Zeitgenossen in seine Architektur des Zacherlpalastes in Wien eine slawische Note eingebracht. Später schuf er zwischen den beiden Weltkriegen in Prag auf Hradčany und in Ljubljana eine einzigartige Kunst, die auf der betont persönlichen Bearbeitung der klassischen Tradition gründete und die in seine Poetik auch die regionalen Charakteristika einbrachte, welche seine Ideen von der Verbundenheit des Nordens und des Mittelmeerraums veranschaulichten. (In seinem Seminar hat er sogar von einer , intuitiven Ahnung" der Verbundenheit der Slowenen mit der Kunst der Etrusker gesprochen.) Sonst wurzelte Plečnik im Geist der Sezession und galt mit seiner Tendenz nach einer ,ewigen Architektur“ (architectura perennis) als eine ahistorische und vor allem nach dem Zweitem Weltkrieg auch unmoderne, wenn nicht explizit retardierte bzw. regressive Erscheinung. In den 80er Jahren des 20. Jahrhunderts wurde er hingegen als einer der Weltvorfahren des Postmodernismus angesehen und popularisiert. So figuriert er heute auf mythischem Niveau sogar als das auffallendste Symbol der slowenischen Schöpferkraft, und einige vergleichen ihn sogar mit Prešeren. Noch vor Plečnik hat seine Spuren in Wien und Ljubljana der Urbanist, Architekt und Denker Maks Fabiani hinterlassen. Fabiani verband die historische Tradition mit der Sezession und den Anfängen der modernistischen Architektur.

Unter den Bildhauern derselben Zeit war in der Generation der akademisch geschulten Schaffenden, die auf die Generation der Bildschnitzer folgten, Fran Berneker, Autor des Trubar-Denkmals in Ljubljana, von besonderer Bedeutung, da er als erster eine moderne Sensibilität bezeugte.

Seither gilt das 20. Jahrhundert in allen Kunstgebieten als die Zeit der Blüte auf europäischem Niveau, das in einer Verbindung der Tendenzen nach Aktualität mit der Suche nach Authentizität, wie auch in der Reflexion über die slowenische Tradition zum Ausdruck kam. So können wir einzelne Erscheinungen nur in einem breiteren europäischen oder sogar Weltkontext sehen und nicht nur, wie einst, vor allem in mitteleuropäisch begrenztem Rahmen deuten. In einem Teil der zeitgenössischen Kritik wurden in bezug auf die Migrationen der stilistischen Impulse auch die Interpretationen des Verhältnisses zwischen der Akzeptanz von sogenannten Einflüssen und dem eigenen individuellen Ausdruck (der auch zum Träger des Nationalen werden konnte) besonders wichtig. 
Nach dem Ersten Weltkrieg haben sich nach dem Zerfall der Österreichisch-Ungarischen Monarchie und wegen der neuen geschichtlichen Umstände nach der Gründung des neuen südslawischen Staats (Staat der Serben, Kroaten und Slowenen, später Jugoslawien genannt) auch die Kunstverbindungen in neuen Richtungen im südslawischen Kontext orientiert. So waren für die slowenische Kunst vor allem die Einflüsse von dem damals weltberühmten Bildhauer Meštrović und seit den 30er Jahren die Rolle der sich an Paris orientierenden Akademie der bildenden Kunst in Zagreb von konstitutiver Bedeutung. Nach dem Zweiten Weltkrieg, im Kontext des neuen jugoslawischen Staates, war wegen der Suche nach einer rituellen Archaik oder dem monumental Heroischen bei einzelnen Künstlern auch die Anknüpfung an die byzantinistische Monumentalität der balkanischen Fresken zu beobachten (bei Marij Pregelj und Jože Ciuha), was von Ausländern wegen ihrer pauschalen Vorstellung von jugoslawischer Kulturtradition irrtümlich als ein Ausdruck der slowenischen historischen Verbundenheit mit der östlichen Kunsttradition gedeutet wurde. Im Zeichen des Exponierens einer (zwar künstlich konstruierten) slowenischen Authentizität ist in der Architektur des Ivan Vurnik (Vurnik organisierte nach dem Ersten Weltkrieg die Gründung der Hohen Schule für Architektur in Ljubljana; in der Architektur war er später ein Protagonist des Funktionalismus) in den frühen 20er Jahren des 20. Jahrhunderts noch eine Tendenz zur Formierung eines slowenischen Architekturstils (das Gebäude der Gegenseitigen Versicherungsanstalt in Ljubljana) zu beobachten; dabei arbeitete mit ihm seine Frau, die Deutsche Helene Vurnik, geborene Kotter, zusammen. Im Geiste der spezifischen kulturpolitischen Umstände sind als Kuriositäten auch die Versuche der Schaffung einer spezifischen slowenischen Authentizität in der Kunst aus Gründen der nationalen Verteidigung entstanden: Nach dem Ersten Weltkrieg vor allem die Malereien von Tone Kralj in den Kirchen des Küstenlands, das damals Italien zufiel (und erst nach dem Zweiten Weltkrieg in die Republik Slowenien eingegliedert wurde).

Gerade die Zeit nach dem Ersten Weltkrieg hat im Zeichen des sogenannten Expressionismus eine Erneuerung der slowenischen Kunst gebracht. Die Kunst des slowenischen Expressionismus basierte auf der mit der religiösexistentiellen und nationalen Symbolik verbundenen Ausdruckskraft. Ihr Wesen war Ausdruck der Ankündigung der Notwendigkeit einer innerlichen Wiedergeburt, gerichtet auf geistige Dimensionen. Die Künstler haben in dieser geistigen Exaltation die formellen künstlerischen Voraussetzungen der Sezession angepasst oder die primären Formen der slowenischen Volkskunst mit den expressionistisch deformierten, auch vom deutschen Expressionismus beeinflussten Formen (die Bewunderer des deutschen Expressionismus waren einige Zeit vor allem die Brüder Drago und Nande Vidmar) und mit den symbolisch gemeinten abstrakten Tendenzen in Verbindung gebracht. Der Maßstab ihrer spezifischen Authentizität ist ihre mit der Ausdruckskraft verbundene Originalität. Eine höchst spezifische Erscheinung war unter ihnen der vielseitige Künstler France Kralj, der, mit der bäuerlichen Tradition in Berührung, in 
den 30er Jahren sogar eine spezifisch slowenische Kunst zu konstituieren versuchte, indem er die mythisierte bäuerliche Ursprünglichkeit wiederzuerwecken trachtete. Als Organisator stellte er, ähnlich wie vor ihm Jakopič, die Kunst seiner Generation auch in Europa, z. B. im Berliner Sturm, aus. In der zweiten Hälfte der 20er Jahre hat sich ein Teil slowenischer Kunst auch ausdrücklich an die Tendenzen der deutschen „Neuen Sachlichkeit“ angeschlossen.

Den Weg zum Expressionismus hat schon Fran Tratnik angedeutet, der sich als ein ausgezeichneter Zeichner in den damals eminentesten europäischen Zeitschriften, z. B. Simplizissimus, einen Namen machte. Mit seinen pessimistisch empfundenen großstädtischen Motiven hat er in unsere Kunst als erster auch das soziale Engagement eingeführt, verbunden mit einer allgemeineren symbolistischen Vorstellung von der Hoffnungslosigkeit der ewig menschlichen Sehnsucht, womit er dem zwar etwas optimistischeren Ivan Cankar nahe stand. Zwei hervorragende expressionistische Künstler waren auch auf der einen Seite der Küstenländler Veno Pilon, der sich in seinem Werk kennzeichnend zwischen der deutschen Exspressivität und dem italienisch geprägten architektonisch gemessenen Plastizismus bewegte, und auf der anderen der Gründer der slowenischen künstlerischen Graphik Božidar Jakac, der seinen Doppelgänger im Kurent, einer mythischen künstlerischen Figur aus einem Buch von Ivan Cankar, fand; später wandte er sich dem künstlerischen Entdecken der slowenischen Landschaft zu. Unter den im Ausland geborenen expressionistischen Malern wurde allmählich Jan Oeltjen in Ptuj heimisch, dessen Schaffen erst kürzlich auch in Deutschland Interesse in seiner Geburtsgegend Oldenburg erweckte.

Das historische Verdienst an der Umorientierung der slowenischen Kunst von den deutschen zu den französischen fauvistischen Einflüssen und Anregungen, womit die Möglichkeiten des künstlerischen Ausdrucks wesentlich erweitert wurden, wird dem Maler Miha Maleš zugeschrieben. Maleš hat mit einer poetischen Verspieltheit die populäre Volkstradition und das kosmopolitische Raffinement mit seinem eigenen persönlichen Lyrismus verbunden. Alle diese nach dem Ersten Weltkrieg tätigen Künstler standen in ihrem inhaltlichen Streben mit den Tendenzen der zeitgenössischen Literatur, vor allem der lyrischen Dichtung, aber auch der anderen künstlerischen Gebiete, in einem engen Kontakt. Heute werden sie manchmal auch mit den „Impressionisten“ verglichen, obzwar die letzteren wegen ihrer historischen Rolle und großen Spannweite ihrer Schaffenszeit noch in der gesamten ersten Hälfte des 20. Jahrhunderts maßgeblich die Vorstellungen von der bildenden Kunst beherrschten. Daher hätten sie, so einige Thesen, vor allem wegen der mythologisierten Verehrung, die man ihrem Schaffen entgegenbrachte, auch die Rezeptionsempfänglichkeit für jene Kunstprinzipen, die mehr die Rolle der Konstruktion betonten, gehemmt. Solche Kunstprinzipien hat als erster Avgust Černigoj aus Triest manifestiert. Černigoj stand in engen künstlerischen Beziehungen mit dem jung verstorbenen Dichter Srečko Kosovel und studierte eine 
Zeitlang als einziger Slowene am deutschen Bauhaus; heute ist er ein Synonym für die Avantgarde. Diese manifestierte sich nach außen am ausdrücklichsten durch die internationale Zeitschrift Tank, von der aber nur zwei (als 1 1/2 und 1 1 $1 / 2-3$ gezeichnete) Nummer erschienen. Später wurden, auch im Kontext des Expressionismus von France Kralj, gewisse, das Kubische betonende Impulse allenfalls im Sinne einer Geometrisierung angenommen, die eine zeitlose Ursprünglichkeit suggerieren sollten.

In den 30er Jahren wandte sich die Malerei im Geist des sogenannten bürgerlichen „farbigen Realismus“ zum ästhetischen Raffinement, mit welchem sie ihre Motive - Landschaften, Figuralik, Stillleben - poetisch oder intimistisch belebte. Teilweise pflegte die Malerei derselben Zeit auch soziales bzw. politisches Engagement. Erst in dieser Zeit sind (dank eines von der Regionalverwaltung ausgeschriebenen Wettbewerbs) auch die ersten ausgesprochenen Werke der sogenannten historischen Malerei mit den Motiven aus der slowenischen Geschichte entstanden, die mit ihrer Motivik unsere historische Existenz seit der Besiedlung zu betonen versuchten (der Wettbewerbgewinner war Gojmir Anton Kos). In der bildenden Kunst herrschte eine symbolische Existenz, mit der sich Slowenen in ihrem kollektiven Empfinden identifizieren konnten und sie zuvor vor allem in der Stimmung der Landschaft gesucht hatten, die in der Zwischenkriegszeit in allen ihren regionalen Varianten, zuletzt auch in Prekmurje, zum Ausdruck kam; das war künstlerisch zwar weniger interessant, dafür aber bereits in folkoristischen Interpretationen bzw. folkloristischer Motivik noch vor der Gründung des Künstlervereins Vesna, etwa im Realismus des späten 19. Jahrhunderts (z. B. bei Ferdo Vesel), zu beobachten.

Während des Zweiten Weltkriegs formierte sich im Kontext des Nationalen Befreiungskampfes als ein im europäischen Kontext spezifisches Phänomen und als Ausdruck eines krampfhaften Willens nach nationaler Selbsterhaltung in der Kunst dieser Jahre die sogenannte „Partisanen-Graphik“ als bedeutendste Erscheinung, die ausdrücklich an das Erbe des Expressionismus anknüpfte (die bedeutendsten Graphiker waren die Partisanen Božidar Jakac, France Mihelič und Nikolaj Pirnat.)

Nach dem Krieg öffnete sich die auf die formalen Ansätze des europäischen modernistischen Schaffens gestützte slowenische Kunst - nach einer sehr kurzen Episode des indoktrinierten sozialistischen Realismus - mit der Gründung der ersten slowenischen Akademie für bildende Kunst in Ljubljana immer intensiver den aktuellen europäischen Kunstströmungen, um sich später auch gegenüber den Einflüssen der eindringenden Amerikanisierung, die am auffälligsten in der Wolkenkratzerarchitektur zum Ausdruck kam, aufnahmefähig zu zeigen.

Im Prozess der Modernisierung der Kunstgriffe, vor allem im Sinne einer mit der Tradition der Karstarchitektur verbundenen, die Konstruktion betonenden Architektonik, spielte Lojze Spacal aus Triest nach dem Krieg die katalysatorische Rolle eines ,zur Welt geöffneten Fensters“. Mit seiner architektonischen Durchsichtigkeit und primären Archaik hat die Konfiguration des 
befreiten Küstenlandes im slowenischen Kontext eine neue, spezifische Bedeutung (z. B. mit dem Graphiker Vladimir Makuc) gewonnen. Die bedeutendsten slowenischen Maler überließen sich auch in der Zeit der Suche nach neuen Formen nicht etwa einem bloß formalistischen Experimentieren, sondern passten verschiedene neuere Kunstgriffe vor allem an eine den Ausdruck betonende Figuralik mit stark hervorgehobenem existentiellem Inhalt an, die auf die archetypische, auf die antiken Mythen sich stützende Motivik wie bei Marij Pregelj einerseits, und auf die persönliche existentielle Erfahrung wie bei Gabrijel Stupica andererseits, gerichtet war. Stupica, unter anderem Autor zahlreicher Selbstbildnisse, hat mit der ängstlichen Stimmung seiner Malerei für einige Denker, die sich mit dem Problem des Slowenentums beschäftigten (Jernej Vilfan), ein eigenartiges Synonym der slowenischen Gehemmtheit, Ungelassenheit und Angst abgegeben. France Mihelič hat das Wesen der slowenischen mythischen Geschichte in der archaischen Verbundenheit mit der Tradition gesehen; dabei stützte er sich auf den zentralen slowenischen heidnischen Mythos des Kurent, der mit der pannonischen Maske des Kurent aus der Ptuj-Gegend verwandt ist. Mit dem Bildnis Der Tod des Kurent hat er in seine Malerei dabei schon früh eine wesentliche Botschaft über die unentrinnbare individuelle Sterblichkeit, die in die Prozesse des unendlichen Wiedergebärens der Natur und in die Grausamkeit der Geschichte eingefangen ist, eingebracht. Diese Idee hat er dann in die phantastische Kunst, die sonst bei den Slowenen etwas Seltenes ist, übertragen. So hat er sich auch im Ausland den Namen eines sehr deutlich als slowenisch zu erkennenden, doch keineswegs folkloristischen, sondern modernen, universal verständlichen Künstlers gemacht. Ein besonders erschütternder Maler mit betont ausgearbeiteter Ausdrücklichkeit seiner Figuralik ist der oben bereits genannte Zoran Mušič, der nach dem Krieg in Paris und Venedig lebte. In seinem Streben nach geistiger Ähnlichkeit mit Stupica oder Mušič hat unter den etwas jüngeren Malern Janez Bernik seine differenziert abstrakten Erfahrungen in einer expressiv-meditativen Figuralik kondensiert. Als eine ganz originelle Erscheinung hat sich der nach seinen frappierenden Bildnissen aus der Pathologie bekannte Jože Tisnikar mit einer „nordischen“ Primarität ausgezeichnet. Der Maler und Priester Stane Kregar, der vor dem Krieg als einziger unter den Slowenen mit seinem Sinn für das Geistige in seine Werke surrealistische Impulse aufgenommen hatte, wandte sich nach dem Krieg sublimierten abstrakten Landschaften zu und erneuerte auch die kirchliche Malerei, vor allem mit zahlreichen Vitragen, die er auch in die alte gotische oder barocke Architektur wunderbar harmonisch einzugliedern verstand.

Infolge der Gründung der internationalen graphischen Biennale in Ljubljana Mitte der 50er Jahre (die Anregung dazu hat Božidar Jakac nach dem Vorbild der graphischen Ausstellungen in Lugano gegeben) kam auch der slowenischen Graphik eine Sonderrolle zu. Mit ihr ist - vor allem dank der technischen Experimente Riko Debenjaks - der Begriff der sogenannten ,graphischen Schule von Ljubljana" verbunden. Unter ihren Repräsentanten hat sich 
der poetische Marjan Pogačnik mit seiner originellen Behandlung der subtilen reliefartigen farbigen Radierung besonders ausgezeichnet und ist auch im Ausland auf großen Widerhall gestoßen; er wurde von allen slowenischen Graphikern in der ausländischen, besonders amerikanischen Fachliteratur am stärksten beachtet. In der Zeit nach dem Krieg entwickelten sich bei uns auch spezielle künstlerische Gattungen, so z. B. die Karikatur (Stane Jagodič). Weltniveau hat die slowenische Bücherillustration für Kinder und Jugend erreicht (Marlenka und Marija Lucija Stupica). In der Kunst kam es auch zu einer neuen Rezeption der Varianten der sogenannten Neoavantgarde, etwa der Formen des Neokonstruktivismus und des Konzeptualismus. Einer der Mitbegründer der bedeutendsten slowenischen konzeptualistischen Gruppe der 60er Jahre war Marko Pogačnik, der sich später mit seiner Meditationserfahrung dem nicht nur künstlerisch konzipierten, weit bekannten Projekt der „Heilung der Erde“ widmete. In der Bildhauerei spielte nach dem Krieg Drago Tršar eine bahnbrechende Rolle, der die Expression seiner Figuren mit dem kollektiven Bild der Menge verschmolz und in die bildhauerische Masse Motive der Urbanisation einbrachte. Wegen der herrschenden Rolle von Jože Plečnik hat sich der Architekturausdruck erst nach dem Krieg moderneren funktionalistischen, häufig unpersönlich unifizierten Formen überlassen; so haben die Wolkenkratzer auch aus ideologischen Gründen, um die Kirchtürme zu überbieten, die Vedute der Hauptstadt Ljubljana gründlich transformiert und meiner Meinung nach auch deformiert. (Wegen der zeitlichen „Verspätung“ der Anfänge des Funktionalismus hat der Kunsthistoriker Stane Bernik für diese Architektur die Bezeichnung ,,der verlängerte Funktionalismus“ in Umlauf gebracht.)

In den 80er Jahren kam es vermutlich auf allen Kunstgebieten und besonders in der Malerei zu einer neuen Expansion der Imagination. Die Malerei dieser Zeit stand im Zeichen der sogenannten ,personellen Poetiken“; dabei war eine neue Differenziertheit, die mitunter mit der geographischen Gliederung zusammenhing, zu beobachten, die von der Kritik auch mit dem Begriff Genius loci bezeichnet wurde. In dieser Perspektive sind die Verinnerlichung und mythische Anlehnung an eine expressive Ursprünglichkeit des nordöstlichen Sloweniens besonders augenfällig (der existentielle Zdenko Huzjan oder der ekstatische Zmago Jeraj); die Verpflichtung dem Mediterranen gegenüber, die mit einer spezifischen mittelalterlichen Archaik auch der palimpsestartigen Malerei von Emerik Bernard ein besonderes Gepräge verleiht, zeugt von einer gelasseneren Lebenslust (Živko Marušič). Die 80er Jahre waren darüber hinaus auch in der Bildhauerei eine Zeit der Blüte; in diesem Kontext sind zwei in Bosnien und Herzegowina geborene Bildhauer, Mirsad Begić und Jakov Brdar (dieser hat eine vielbeachtete Ausstellung im Pergamon-Museum in Berlin gehabt und ist auch Schöpfer des ersten einem Slowenen, dem General und Dichter Rudolf Maister, gewidmeten Reiterstandbilds in Ljubljana), von besonderer Bedeutung, die zu jenen in Ljubljana ausgebildeten, nicht aus Slowenien stammenden Künstlern gehören, die das Profil der slowenischen Kreativität in letzten Jahrzehnten maßgeblich ergänzen. Im Kontext der Erneuerung 
der Avantgarden und des Manifestierens der politischen Konstellationen des slowenischen Territoriums zwischen Ost und West haben größte internationale Beachtung die Manifestationen der sogenannten ,retrogardistischen“ Malergruppe IRWIN erreicht. Diese Malergruppe ist eine Abteilung der Gruppe Neue slowenische Kunst, die trotz des provokativ deutsch geschriebenen Namens in ihr absichtlich eklektisches Programm auch die nationalen "Ikonen“ der slowenischen bildenden Kunst aufgenommen hatte, so z. B. in der Ausstellung Slovenske Atene [Slowenisches Athen].

Noch später, eigentlich bis zum heutigen Tag, ist neben der Erneuerung der früheren Kunstformen, vor allem des Informels und des Konzeptualismus, in der slowenischen Kunst eine zunehmende Verwendung von neuen technologischen, vor allem elektronischen Medien zu beobachten; dabei wird die bildende Kunst zunehmend in einen engagiert oder bloß kulturologisch gefärbten Ausdruck der globalen Erscheinungen in der Gesellschaft verwandelt. So wird das heutige Verständnis der Kunsttätigkeit, die als solche fast jede visuell lesbare Äußerung bzw. ein in einen Galerieraum oder in urbanes Exterieur lokalisiertes Geschehen in ihren Horizont einbezieht, immer häufiger der Macht der Trends und der richtunggebenden Rolle der Kustoden untergeordnet, zumal auf dem Gebiet der sogenannten ,alternativen Kultur“, die in der Tat meist eine aus sozialen Gründen gesellschaftlich geförderte (und tatsächlich in die etablierte Kulturpolitik völlig integrierte) Tätigkeit ist.

Da das Territorium des slowenischen Staates klein, andererseits aber das Bewusstsein von der Rolle der Kultur sehr ausgeprägt ist (deswegen hat schon der Literaturkritiker Josip Vidmar in der Zeit vor dem Zweiten Weltkrieg die Perspektive Sloweniens in einer höchst qualitativen Kulturentwicklung und Transformation Sloweniens zum „,neuen, slowenischen Athen“ gesehen), ist die Kunsttätigkeit in Slowenien heute quantitativ sehr saturiert und polyzentrisch, dabei aber auch für ausländische Kunstkenner und -liebhaber, vor allem jene aus unserer Nachbarschaft, interessant. Mit der Zahl der wirklich bedeutenden Künstler verglichen, ist die Zahl der registrierten Künstler naturgemäß sogar drastisch unproportioniert; doch schon diese ungewöhnliche quantitative Konzentration der Protagonisten und der Ausstellungen dürfte mitunter zu einer kulturellen Grundlage werden, die auch die Entwicklung bedeutenderer Erscheinungen fördern kann.

Als Ganzes besehen, bietet die slowenische Kunst auch einen Einblick in historische Verwandlungen der slowenischen Kultur in Vergangenheit und Gegenwart, in verschiedene Möglichkeiten des Kunstschaffens und in die Wahl, Rezeptionsweisen und Adaptierung allerlei künstlerischer Impulse oder Einflüsse; dabei jedoch auch in die slowenischen Spezifika, die vor allem in der Individualität der einzelnen Künstler zu suchen sind. Die bildende Kunst ist mit dem Universalismus ihrer Sprache auch da, wo es um spezifische Zusammenhänge mit den Charakteristiken des Territoriums geht, allerdings mehr von der Struktur des breiteren Kontextes bedingt als von nationalen Spezifika. Auch die bildende Kunst setzt sich jedoch - mit zunehmender Rolle des Ex- 
ponierens der persönlichen Authentizität - in ihrem Inhalt mit denselben Problemen auseinander, die in der Literatur (und da vor allem in der Poesie, die bei Slowenen das populärste Kunstmetier ist) hervorgehoben werden, und somit auch mit denjenigen, die manchmal für typisch slowenisch gehalten werden. Die Bezeichnungen solcher Probleme sind in der bildenden Kunst durch die Labyrinthe des menschlichen Schaffens mit nicht zu fassenden Zeichen eingeschrieben, die man sich bisher als slowenischen, von der Sehnsucht getragenen Lyrismus oder Schmerz (diesen hat Kunsthistoriker Tomaž Brejc im Kontext der Malerei des späten 19. und des 20. Jahrhunderts sogar mit dem spezifischen Terminus „dunkler Modernismus“ in Verbindung gebracht), als Hemmung oder Misstrauen gegenüber den Extremen, als Sinn für das „gewerbliche" Abschließen und dementsprechenden Mangel an Großzügigkeit oder als vergeistigte Verlängerungsweise des figuralen Proportionierens zu lesen oder zu entziffern (aber auch zu verneinen) bemüht hat.

Den heutigen historischen Zeitpunkt des Übergangs, in dem die neuen Verbindungen und Integrationen des historischen Europas stattfinden, vergleichen einige in Slowenien mit der Situation um die Zeit der Festigung der Macht des universalistischen Christentums in Europa, das damals auch die Slowenen in die neuen Kulturströmungen einbezogen hat und von entscheidender Bedeutung auch für die spätere slowenische Geschichte war. Eben jene historische Periode wurde im Zeichen eines differenziert interpretierten Dilemmas von der Treue gegenüber der heidnischen altslawischen Tradition einerseits und von dem sich anbahnenden Christentum andererseits vom Dichter France Prešeren in seiner bereits erwähnten änigmatischen epischen Dichtung Krst pri Savici im Kontext der in seiner Zeit aktuellen ethischen und nationalen Fragen thematisiert. Das Bewusstsein von der Notwendigkeit einer verantwortungsvollen Erhaltung und weiteren Entwicklung der nationalen Attribute (die bisher vor allem auf der Grundlage der von Primož Trubar in der Reformation erschaffenen Literatursprache auch in anderen Gebieten vorwiegend im 19. Jahrhundert erfolgte - allerdings mehr als ein Resultat der Spontaneität und vor allem dank der kreativen Energien, mit welchen sich die Slowenen auf die Kulturkarte Europas gesetzt haben) scheint heute im Vergleich zu jenen Zeitabschnitten, in denen das Nationalbewusstsein noch nicht formiert und daher vor allem im Identifikatorischen der Sprache impliziert war, umso entscheidender, bedeutungs- und anspruchsvoller, obgleich dieses Nationalbewusstsein und Selbstverständnis heute in Slowenien nicht für alle auch etwas Selbstverständliches ist.

Über die Zukunft der slowenischen Kultur nach einer erneuten Überwindung von Grenzen (davon ist nach Meinung einiger slowenischer Historiker vielleicht eine Situation zu erwarten, die in einem gewissen Maße der Erfahrung der Lage in der einstigen multinationalen Österreichisch-Ungarischen Monarchie entsprechen könnte; das würde für das nationale Interesse auch eine neue Gefahr bedeuten) wird allerdings - neben allen pragmatischen $\mathrm{Zu}$ und Umständen einer solchen Tat - auch die Verpflichtung der Slowenen zur 
eigenen geistigen Schaffenskraft entscheiden. Das bedeutet vor allem ein intensives Verhältnis zu jenen lebendigen produktiven Kräften, die die slowenische Kultur und Kunst die ganze Zeit hindurch formierten und prägten und die auch für ihre Zukunft entscheidend sein werden. Es geht hier also auch um die Bereitschaft von uns Slowenen, unsere Vergangenheit, mit der wir selbstverständlich in den europäischen Kulturkontext gestellt sind, in einer spezifischen Perspektive zu sehen, die sich zum Ausgangspunkt das Slowenentum, wie es aus dem Bedürfnis der nationalen Selbstaffirmation in der Wiedergeburt der slowenischen Kunst im 20. Jahrhundert formuliert wurde, genommen hatte. Diese Perspektive schließt jedoch den Blick, der vor allem in die Kulturbeziehungen (in allen ihren Richtungen und Nuancen) im europäischen Kontext gerichtet ist, keineswegs aus; damit ist nicht etwa eine Abgeschlossenheit der Kunst oder ihre Volkstümlichkeit gemeint, sondern ein Versuch, in der bildenden Kunst der Vergangenheit und Gegenwart auch die Beziehungen zur historisch erfassten Problematik der nationalen Authentizität und eventueller Besonderheiten auf höchstem künstlerischem Niveau zu entdecken und zu interpretieren.

\section{Literaturverzeichnis}

Brejc, Tomaž, 1991: Temni modernizem. Slike, teorije, interpretacije. Ljubljana.

Cevc, Emilijan, 1966: Slovenska umetnost. Ljubljana.

Čopič, Špelca, 1966: Slovensko slikarstvo. Ljubljana.

Golob, Nataša (Hrsg.), 1998: Umetnost na Slovenskem. Od prazgodovine do danes. Ljubljana.

Komelj, Milček / Kranjc, Igor (Hrsg.), 1986: Ekspresionizem in nova stvarnost na Slovenskem. Ljubljana.

Komelj, Milček, 1992: „Slovenska samobitnost v luči likovne umetnosti“, in: XXVIII. seminar slovenskega jezika, literature in kulture. Zbornik predavanj. Ljubljana, 161-178.

Komelj, Milček, 1997: Poteze / Striche. Slovensko slikarstvo v 20. stoletju / Slowenische Malerei im XX. Jahrhundert. Ljubljana.

Mikuž, Jure, 1995: Slovensko moderno slikarstvo in zahodna umetnost. Od preloma s socialističnim realizmom do konceptualizma. Ljubljana.

Molè, Vojeslav, 1965: Umetnost južnih Slovanov. Ljubljana.

Prelovšek, Damijan, 1995: „Identität und Nachholbedarf“, in: Bürgerliche Selbstdarstellung; Bürgertum und Habsburgermonarchie, 4. Wien. Köln. Weimar.

Prelovšek, Damijan, 1997: Jože Plečnik 1872-1957. New Haven. London.

Stelè, France, 1924: Oris zgodovine umetnosti pri Slovencih. Kulturnozgodovinski poskus. Ljubljana.

Stelè, France, 1935: Monumenta artis Slovenicae I. Ljubljana.

Stelè, France, 1935: Monumenta artis Slovenicae II. Ljubljana.

Stelè, France, 1960: Umetnost v Primorju. Ljubljana.

Stelè, France, 1970: Slovenski impresionisti. Ljubljana. 
Šumi, Nace, 1975: Pogledi na slovensko umetnost. Ljubljana.

Vilfan, Jernej, 1985: Strupena nevesta (likovne beležke). Maribor.

Zadnikar, Marijan, 1970: Romanska umetnost. Ljubljana.

Žitko, Sonja, 1989: Historizem v kiparstvu 19. stoletja na Slovenskem. Ljubljana. 
